\title{
Thrombocytopenia and Carcinoma
}

\author{
G. N. BRODIE, ${ }^{*}$ M.B., M.R.A.C.P. ; D. BLISS, $\dagger$ M.B., M.R.A.C.P. ; B. G. FIRKIN, $\ddagger$ M.B., F.R.A.C.P.
}

British Medical fournal, 1970, 1, 540-541

$S^{\text {un }}$ ummary: The cases of two patients suffering from carcinoma of the lung and severe thrombocytopenia are described. The thrombocytopenia was probably due to an immune mechanism and not to marrow replacement by carcinoma or consumption coagulopathy. It is suggested that patients in the older age group presenting with thrombocytopenia may have occult carcinoma.

\section{Introduction}

Immune thrombocytopenia has been recorded as occurring during the course of a great variety of conditions. Its reported relation to neoplastic disease has so far been limited to leukaemias and lymphomas, most notably chronic lymphocytic leukaemia (Baldini, 1966). Harrington et al. (1956) mentioned carcinomatosis in a list of causes of secondary thrombocytopenia. Three cases were recorded and a platelet agglutinin was found in one of them. No other details of the cases were recorded, and so far as can be determined there have been no reports of immune thrombocytopenia complicated by carcinoma.

This report draws attention to two patients who were suffering from thrombocytopenia and carcinoma and in whom the thrombocytopenia was due to some basis other than marrow replacement. One of them had a grossly shortened platelet survival, and an active search for a consumption coagulopathy was unsuccessful. In the other case necropsy showed no evidence of fibrin or platelet deposition in the small vessels.

It is suggested that some instances of thrombocytopenia associated with carcinoma may be on an "immune" basis and should not be attributed to either marrow replacement or coagulation pathology without adequate documentation.

\section{Case 1}

A man aged 72 was referred to hospital for further investigation of thrombocytopenia which had been present for two years. Symptoms had been limited to occasional bruises on the forearm. He had smoked 10 cigarettes a day for 50 years and had suffered from recurrent bronchitis over this time. There was no history of recent chest pains, cough, or haemoptysis, but he had lost $28 \mathrm{lb}$. (12.7 kg.) in weight during the six months before his admission.

Physical examination disclosed moderate bruising of both forearms. There was no splenomegaly. A chest $x$-ray film showed a solid lesion in the right costophrenic angle. The haemoglobin was 13.0 g. $/ 100 \mathrm{ml}$., W.B.C. $8,500 / \mathrm{cu}$. mm., platelet count $10,000 /$ cu. $\mathrm{mm}$. and reticulocyte count $1 \%$. Bone marrow aspiration showed increased numbers of megakaryocytes, myeloid hyperplasia, and evidence of iron deficiency. Platelet life-span, with isologous platelets labelled with ${ }^{51} \mathrm{Cr}$, was 1.5 days (normal 7 to 10 days; O'Neill and Firkin, 1964).

A right lower lobectomy was performed and an anaplastic carcinoma removed. Metastatic carcinoma in the hilar lymph nodes was removed at surgery. After removal of the tumour the platelet count rose to $119,000 / \mathrm{cu}$. $\mathrm{mm}$., but over the next three weeks it fell to its pre-operative level. A platelet survival carried out one month postoperatively was 14 hours, the platelet count at this time being $45,000 / \mathrm{cu}$. mm. The partial thromboplastin time was 36 seconds, prothrombin index $100 \%$, thrombin time 10 seconds, plasma

*Research Fellow, Clinical Research Unit, Royal Prince Alfred Hospital, Camperdown, N.S.W.

†Medical Registrar, Repatriation General Hospital, Concord, N.S.W.

$\ddagger$ Professor of Medicine, Monash Medical School, Alfred Hospital, Prahran, Victoria. fibrinogen $295 \mathrm{mg} . / 100 \mathrm{ml}$, and fibrin split products were not demonstrable by the haemagglutination technique. He has continued to remain thrombocytopenic with platelet counts around $30,000 / \mathrm{cu} . \mathrm{mm}$.

\section{Case 2}

The patient, a man aged 56, noticed the onset of spontaneous petechial haemorrhages over both arms in October 1965 and was admitted to another hospital where the platelet count was found to be $20,000 / \mathrm{cu}$. mm. Six weeks later a solid, irregular lesion in the left upper zone of the chest was discovered on $x$-ray examination. One week later excessive bleeding was encountered following dental extractions. During this time he had noticed occasional night sweats, but no other symptoms were present. On examination clubbing of the fingers, dullness and diminished breath sounds over the left upper zone of the chest anteriorly, and purpuric spots over the arms, legs, and trunk were found.

Investigations in January 1966 showed: haemoglobin 12 g./100 ml., W.B.C. $7,000 / \mathrm{cu}$. mm. with a normal differential, platelets $15,000 / \mathrm{cu}$. mm., and E.S.R. $15 \mathrm{~mm}$./hour. The reticulocyte count was less than $2 \%$. The Duke bleeding-time was greater than 30 minutes, the Lee and White clotting-time was seven minutes, and clot retraction was absent. Marrow aspiration showed a normocellular marrow with plentiful particles and an increased number of megakaryocytes. No other abnormality was detected. The prothrombin time was normal and L.E. cell preparations were negative.

Sputum cytology showed adenocarcinoma cells. Prednisone therapy was tried over a five-week period in a dosage range of $\mathbf{4 0}$ to $100 \mathrm{mg}$./day. There was no response in his platelet count.

On 2 February splenectomy and left radical pneumonectomy was performed. There was a large carcinoma of the left upper lobe with extensive mediastinal metastases. The spleen was normal in size, weighed $205 \mathrm{~g}$. and was microscopically normal. Microscopical examination of the tumour confirmed the diagnosis of adenocarcinoma. There was no change in the platelet count after surgery. Evidence that an adequate splenectomy had been performed was proved by the finding of Howell-Jolly bodies and target cells in the peripheral blood.

The patient remained thrombocytopenic throughout the remaining nine months of his life and in November 1966 suffered a subarachnoid haemorrhage from which he died.

Necropsy showed metastatic carcinoma in the mediastinum, liver, and kidneys. There was extensive subarachnoid haemorrhage, no local site for the bleeding being found. No tumour was found in sections of bone marrow, and no evidence of fibrin or platelet deposition in vessels was noted in any of the tissues examined.

\section{Discussion}

These two patients have features in common which made us consider that their thrombocytopenia was of an immune nature. The bone marrows contained normal or increased numbers of megakaryocytes. The thrombocytopenia was of a severe degree, and in one the platelet survival time was less than 24 hours. It has been the experience of several investigators in this field that platelet survivals as short as this are almost invariably found in acute immune thrombocytopenic states (Najean et al., 1963; Baldini, 1966).

Several other possible mechanisms for the development of thrombocytopenia in these patients were considered. Marrow replacement by carcinoma was ruled out on the grounds that there was consistently no morphological evidence for this, both in bone marrow aspirates and in necropsy marrow ma- 
terial. Neither of these patients had a leucoerythroblastic blood picture, and marrow replacement would not be associated with shortened platelet survival.

Microangiopathic changes were not seen in the peripheral blood smears in either patient. Evidence for a haemolytic anaemia was not observed, nor was there any small-vessel involvement by carcinoma in the patient who came to necropsy. Hence, microangiopathic haemolytic anaemia with thrombocytopenia or a consumption coagulopathy would seem unlikely, but the latter was completely excluded only in Case 1, where the fibrinogen level and thrombin and prothrombin times were normal, and no split products were found. No evidence for any other cause of immune thrombocytopenia was found. Neither patient had been exposed to drugs or chemicals likely to cause thrombocytopenia. L.E. cell preparations were negative, and there was no history of a recent viral infection. Lymphomas and leukaemias were not present.

The fact that neither patient responded to splenectomy and/or steroids does not detract from the diagnosis of thrombocytopenia on an immune basis. Baldini (1966) found in dogs that as the titre of antiplatelet antibodies rises platelets became sequestered in the liver as much as in the spleen and the response to steroids and to splenectomy lessens. Hence, it is postulated that these patients either had a very high titre of antibody or there could be an increase in macrophage activity in the reticuloendothelial system (Firkin et al., 1969). The response to splenectomy or steroids in chronic adult idiopathic thrombocytopenic purpura does not appear to bear any direct relation to the presence or absence of demonstrable splenic sequestration or the degree of reduction of platelet survival (Castaldi and Firkin, 1963; Shulman et al., 1964; Baldini, 1966).

Case 1 showed a very transient rise in platelet count after removal of the tumour. Metastatic carcinoma, however, was present in lymph nodes removed at operation, and it is probable that residual carcinoma remains and could explain the subsequent relapse.

The association of neoplasia with thrombocytopenia may well be due to chance, but it seems important to examine thrombocytopenia in neoplastic states more closely in the future and not to assume that it is due simply to bone marrow replacement by the tumour or a consumption coagulopathy. The possibility that patients in the older age group presenting with thrombocytopenia may have an occult carcinoma should also be remembered by the clinician.

REFERENCES

Baldini, M. (1965). New England fournal of Medicine, 274, 1245, 1301, 1360

Castaldi, P. A., and Firkin, B. G. (1963). Australasian Annals of Medicine, 12, 333 .

Firkin, B. G., Wright, R., Miller, S., and Stokes, E. (1969). Blood, 33, 240.

Harrington, W. J., Minnich, V., and Arimura, G. (1956). Progress in Hematology, i, 166.

Najean, Y., Ardaillou, N., Caen, J., Larrieu, M. J., and Bernard, J. (1963). Blood, 22, 718.

O'Neill, B., and Firkin, B. G. (1964). Fournal of Laboratory and Clinical Medicine, 64,188 .

Shulman, N. R., Marder, V. J., Hiller, M. C., and Collier, E. M. (1964). Progress in Hematology, 4, 222.

\section{Preliminary Communications}

\section{Active Immunotherapy with Corynebacter- ium parvum and Chemotherapy in Murine Fibrosarcomas}

British Medical fournal, 1970, 1, 541-544

\begin{abstract}
Uummary: Corynebacterium parvum used alone to en$\checkmark$ hance immunological reactivity produced transient inhibition of the growth of chemically induced isogenic mouse tumours. Attempts were made to combine C.Parvum with cyclophosphamide to see whether this would increase the latter's effectiveness in inhibiting early but established tumours. Of the various regimens tested, the administration of the C. parvum 12 days after a single dose of chemotherapy produced dramatic inhibition of tumour growth and resulted in complete and lasting regressions in up to $70 \%$ of tumour-bearing animals. The most important variable in this regimen is the time between the chemotherapy and the subsequent immunotherapy.

It is possible that non-specific active immunotherapy with agents such as $C$. parvum may be a valuable adjunct to the conventional cyto-reductive treatments of cancer, but the time of administration of such therapy is probably critical for each tumour and for each chemotherapeutic regimen.
\end{abstract}

\section{INTRODUCTION}

The potential value of immunological methods in the control of cancer has received much attention. It is widely held that though such methods are unlikely to lead to the eradication of extensive malignant disease they may be of value as an adjunct to other therapeutic techniques. There is some experimental evidence to support this suggestion. Haddow and Alexander (1964) demonstrated that rat sarcomas are radiosensitized by autoimmunization of the host with irradiated tumour. Glynn et al. (1969) used a combined immunochemotherapeutic system to eradicate Moloney-virus-induced lymphomas in mice. They combined cyclophosphamide with adoptive immunotherapy using tumour-immune lymphocytes and showed that, though neither the chemotherapy nor the immunotherapy used alone was effective, in combination they eliminated the tumours in a significant proportion of treated animals.

Agents which produce a generalized increase in immunological reactivity have been used as a form of non-specific active immunotherapy. Zymosan (Bradner et al., 1958), B.C.G. (Old et al., 1959), and Corynebacterium parvum (Woodruff and Boak, 1966) have induced significant host-mediated inhibition of several types of experimental tumour. The exciting results obtained by Mathé (1969) in the treatment of acute lymphoblastic leukaemia in children suggest that such agents may be usefully combined with the more conventional forms of chemotherapy. Sokoloff et al. (1961) examined the antitumour activity of mitomycin $\mathrm{C}$ and indicated that the oncolysis of several non-specific murine tumours produced by this antibiotic can be enhanced by the use of zymosan. The adjuvant activity of this yeast extract was exquisitely dependent on time of administration, and under some circumstances it merely aggravated the toxic effects of mitomycin C. Martin et al. (1964) also used zymosan, combining it with both cyclophosphamide and surgery in the treatment of spontaneous mammary adenocarcinomas in mice. They concluded that zymosan appreciably enhanced the therapeutic effects of the surgery-chemotherapy combination. They also showed that the 